\title{
Hepatotoxicity in Rats Induced by Aqueous Extract of Polygoni Multiflori Radix, Root of Polygonum multiflorum Related to the Activity Inhibition of CYP1A2 or CYP2E1
}

\author{
Deng-Ke Li, Jing Chen, Zhen-Zhen Ge, and Zhen-Xiao Sun \\ School of Chinese Materia Medica, Beijing University of Chinese Medicine, 6 Wang Jing Zhong Huan Nan Lu, \\ Chaoyang District, Beijing 100102, China
}

Correspondence should be addressed to Zhen-Xiao Sun; sunzxcn@hotmail.com

Received 1 February 2017; Revised 28 March 2017; Accepted 5 April 2017; Published 24 May 2017

Academic Editor: Antonella Di Sotto

Copyright (C) 2017 Deng-Ke Li et al. This is an open access article distributed under the Creative Commons Attribution License, which permits unrestricted use, distribution, and reproduction in any medium, provided the original work is properly cited.

\begin{abstract}
The objective of this study is to investigate the relationship between the hepatotoxicity induced by Polygoni Multiflori Radix (PMR, root of Polygonum multiflorum Thunb., He Shou Wu) and the activity of CYP1A2 or CYP2E1 in the rat liver. Levels of rat serum transaminases ALT and AST were not altered but the activity of CYP1A2 or CYP2E1 in the rat liver was significantly inhibited after oral administration of aqueous extract of PMR under the experimental dosage. However, levels of ALT and AST were significantly increased and the activity of CYP1A2 or CYP2E1 was significantly decreased after injection of specific inhibitor for CYP1A2 or CYP2E1 combined with oral administration of aqueous extract of PMR, especially under the repeated treatment over interval times. Liver histopathological observation showed that a moderate liver injury occurred in rats receiving PMR treatment with the activity of CYP1A2 or CYP2E1 inhibited, but there was no significant liver damage in rats receiving PMR treatment or CYP inhibitor alone. These suggested that low level activity of CYP1A2 or CYP2E1 from genetic polymorphism among people might be one of the important reasons for the hepatotoxicity induced by PMR in clinical practice.
\end{abstract}

\section{Introduction}

Polygoni Multiflori Radix (PMR, root of Polygonum multiflorum Thunb., He Shou Wu) is a traditional Chinese medicine (TCM) that has been used in Chinese clinics for centuries. According to the Pharmacopoeia of the People's Republic of China, the function of PMR is detoxication, eliminating carbuncle, and lubricating the bowels for clinical treatment of sore, carbuncle, scrofulosis, rubella, pruritus, body deficiency in chronic malaria, and constipation [1]. PMR is used widely in China now for different clinical applications such as treating premature graying hair, antiaging, and antihyperlipidemia [2]. Extracts of PMR could completely reverse the C57BL/6 mice hair decolorization induced by $\mathrm{H}_{2} \mathrm{O}_{2}$ with the expressions of $\alpha$-melanocytestimulating hormone ( $\alpha$-MSH) and melanocortin 1 receptor (MC1R) and tyrosinase (TYP) upregulation [3]. As PMR was proved to inhibit the activity of acetylcholinesterase (AChE) and 2,3,5,4' -tetrahydroxystilbene-2-O- $\beta$-D-glucopyranoside
(THSG) and emodin-8-O- $\beta$-D-glucoside (EG) as the main components in PMR were proved to decrease AChE activity and THSG increased the expression of protein phosphatase2A (PP-2A) and microtubule associated protein-2 (MAP-2) in the hippocampus of model rats, PMR was suggested to have the potential for antiaging such as Alzheimer's disease treatment $[4,5]$. PMR polysaccharide was reported to have a significant antihyperlipidemic effect in hyperlipidemic mice by its administration at doses of 50 to $200 \mathrm{mg} / \mathrm{kg}$ BW for 28 days. The results showed that the serum levels of TC, TG, and AI were significantly decreased, whereas the HDL-C, LPL, HL, and LA levels were significantly increased [6].

Hepatic adverse effects have been frequently reported since PMR is widely used in China and other countries $[7,8]$. A metabolomic study on idiosyncratic liver injury induced by different extracts of PMR in rats showed that PMR ethylacetate extract results in evident liver injury, indicated by the significant elevation of plasma alanine aminotransferase and aspartate aminotransferase activities, as well as obvious 
liver histologic damage. PMR ethylacetate extract had close association with the idiosyncratic hepatotoxicity of PMR and provided a metabolomic insight into idiosyncratic HILI (herb induced liver injury) of different extracts from PMR [9]. RUCAM (Roussel Uclaf Causality Assessment Method) or later also synonymously CIOMS (Council for International Organizations of Medical Sciences) was developed to cope with the shortcomings inherited in the causality assessment of drug induced liver injury (DILI). It is well validated by cases with positive reexposure tests serving as a gold standard. Most importantly, RUCAM is a means of assigning points for clinical, biochemical, and serologic features as well as searching for nondrug causes. There is an update of RUCAM as a development of diagnostic methods and sensitive biomarkers, which made a major step forward to facilitate causality assessment in suspected DILI and HILI cases [10]. One of the systematic reviews on PMR related liver injury reported that a total of 12307 inpatients with liver dysfunction and DILI were screened, which included records of 302 military hospitals in China for the past 10 years. DILI patients, who had taken TCM containing PMR, were assessed by RUCAM scale, showing that $22.5 \%$ of the cases are highly probably related to He Shou Wu (RUCAM points $>8$ ), $37.5 \%$ of the cases are probably related (RUCAM points $6-8$ ), and $32.5 \%$ of the cases are possibly related (RUCAM points 3-5) [11]. Levels of alanine aminotransferase (ALT), aspartate aminotransferase (AST), and alkaline phosphatase (ALP) in serum are the main diagnostic markers for HILI and can be considered the current "golden standard" for initial diagnosis and surveillance [12]. An intrinsic form of HILI such as germander (Teucrium chamaedrys) hepatotoxicity is a typical liver injury of the intrinsic form, since it is dose dependent and reproducible in mice [13]. The pathophysiology of idiosyncratic HILI in humans is difficult to assess due to the lack of experimental reproducibility and hence absence of an experimental animal model of HILI. Kava hepatotoxicity is an idiosyncratic liver injury linked to metabolic aberration in unusually susceptible humans, providing an overall low incidence of kava hepatotoxicity in the normal population [14]. We previously reviewed the PMR related acute liver injury in clinical applications in China [15]. It seems that PMR related clinical hepatotoxicity is family related and recurring, suggesting that PMR induced hepatotoxicity might be related to idiosyncratic reaction of patients. Metabolite idiosyncratic DILI is well known to associate with the genetic polymorphisms of liver cytochrome P450 enzyme (CYP450) [16-18].

Polymorphism of CYP includes the lack of CYP isoforms, loss of inducibility, or synthesis of a CYP form with altered catalytic activity. Polymorphisms have been reported to be related to hepatotoxicity of some drugs in the affected individuals $[19,20]$. CYP1A2 and CYP2E1 mainly exist in the liver, accounting for $13 \%$ and $7 \%$ of total CYP450 enzymes, respectively [21]. In addition, CYP1A2 and CYP2E1 exhibit genetic polymorphism in the population [22, 23]. At least 23 allele genes of CYP1A2 have been found so far, and the major mutants are CYP1A2* $1 \mathrm{C}, \mathrm{CYP} 1 \mathrm{~A} 2{ }^{*} 1 \mathrm{D}$, CYP1A2*1F, CYP1A2*1K, CYP1A2*7, and CYP1A2*11. The allele frequency of CYP1A2* $1 \mathrm{C}$ with decreased CYP1A2 activity is approximately 0.22 to 0.25 in the Chinese population [24]. Moreover, CYP1A2 and CYP2E1 are highly conserved among species with $80 \%$ identical nucleic acid sequence between humans and rats [25]. The amino acid sequence of CYP1A2 and CYP2E1 in humans and rats has $70 \%$ [26] and 80\% [27] similarity, respectively. According to a recent study on PMR related hepatotoxicity and genetic polymorphisms of CYP1A2 in clinical patients, the frequency of the CYP1A2 ${ }^{*} 1 \mathrm{C}$ allele is $46.5 \%$ in PMR induced DILI patients, which is significantly different from the frequency of 27.9\% observed in healthy people, indicating that the increase of frequency of CYP1A2* $1 \mathrm{C}$, which decreased the activity of CYP1A2, may be related to the acute liver injury induced by PMR [28]. Recently, we found that enzymatic activity and mRNA expression of CYP1A2 and CYP2E1 in rat liver were significantly inhibited by the aqueous extract of PMR [29], suggesting that the combination of genetic polymorphisms and PMR may induce DILI in rats. This work aimed to investigate whether acute liver injury will occur when PMR is orally administrated to rats with liver CYP1A2 or CYP2E1 inhibited.

\section{Materials and Methods}

2.1. Reagents. PMR was purchased from Tong Ren Tang Technologies Co. Ltd. THSG (batch number: 140317), emodin (batch number: 140422), EG (batch number: 140822), and physcion (batch number: 140211) were purchased from Sichuan Weikeqi Biological Technology Co., Ltd. Cimetidine for injection (Jiangsu Shenlong Pharmaceutical Co., Ltd.; standard: $2 \mathrm{~mL}, 200 \mathrm{mg}$ per ampoule, batch number: 070314) and trans-1,2-dichloroethylene were purchased from Sigma Company (product number: C62209-5G).

2.2. Preparation of the Aqueous Extract of PMR. PMR was decocted twice in $10 \mathrm{x}$ volume of water for 2 hours and $8 \mathrm{x}$ volume of water for 1.5 hours, respectively. The extract was merged together and concentrated using rotary evaporators. Then, it was frozen at $-80^{\circ} \mathrm{C}$ and lyophilized using vacuum freezing drying oven. The powder was dissolved in water at a concentration equal to $5.0 \mathrm{~g} / \mathrm{mL}$ PMR for each experiment. The aqueous extract of PMR is used since water decoction was the most commonly used dosage form for PMR in clinical application.

2.3. High Performance Liquid Chromatography (HPLC) Analysis. The HPLC system was a Waters 1525 instrument with a UV detector (Waters). Separation was carried out on a Dikma Diamonsil C18 column $(5 \mu \mathrm{m}, 250 \times 4.6 \mathrm{~mm})$ at room temperature. A gradient elution program was conducted for chromatographic separation with mobile phase A ( $0.5 \%$ acetic acid) and mobile phase B (acetonitrile) as follows: $0-45 \mathrm{~min}(10 \%-35 \% \mathrm{~B}), 45-65 \mathrm{~min}(35 \%-100 \% \mathrm{~B})$, and $65-70 \mathrm{~min}(100 \% \mathrm{~B})$. The mobile phase was delivered at $1.0 \mathrm{~mL} / \mathrm{min}$. The injection volume was $10 \mu \mathrm{L}$ and the total analysis time was $70 \mathrm{~min}$ for each run. The detection wavelength was $280 \mathrm{~nm}$. The powder of PMR and the standard were dissolved with methanol. All tested solutions were filtered through $0.45 \mu \mathrm{m}$ membrane syringe filters before 
use. The method followed [30], and the calibration curve was established for the standards: the concentration range of THSG from 46 to $920 \mu \mathrm{g} / \mathrm{mL}(Y=29722 \times X-125033$, $\left.r^{2}=0.9998\right)$, the concentration range of EG from 1.04 to $41.6 \mu \mathrm{g} / \mathrm{mL}\left(Y=52836 \times X+12471, r^{2}=0.9992\right)$, the concentration range of emodin from 0.273 to $7.8 \mu \mathrm{g} / \mathrm{mL}(Y=$ $\left.66516 \times X+9285, r^{2}=0.9996\right)$, and the concentration range of emodin methyl ether from 0.111 to $28.86 \mu \mathrm{g} / \mathrm{mL}(Y=67576 \times$ $\left.X-313, r^{2}=0.9998\right)$, and the measurement accuracy and precision are favorable, $\mathrm{RSD}<5 \%$.

2.4. Animals and Treatment. Male Sprague-Dawley (SD) rats $(200 \pm 10 \mathrm{~g})$ were purchased from the Animal Science Center (Peking University Health Science Center, Beijing, China) (production certificate number: SCXK (JING) 20110012). The dose of PMR for animal treatment is based on previous animal experiments, which found that receiving an oral administration of PMR at low dose $(20 \mathrm{~g} / \mathrm{kg} \mathrm{BW})$ or high dose $(40 \mathrm{~g} / \mathrm{kg} \mathrm{BW})$ for 60 days did not affect rat serum ALT or AST levels significantly [31]; the effect of CYP450 level was not taken into account in the previous animal experiments. If the level of CYP450 has an effect on PMR related rat liver injury, a high dose may show a more pronounced effect, and the high dose was adopted in this study. Thirty SD rats were randomly divided into six groups (Figure 1): (a) control group, in which the rats received an oral administration of water; (b) PMR group, in which the rats received an oral administration of PMR of $40 \mathrm{~g} / \mathrm{kg}$ body weight (BW); (c) CYP1A2 inhibitor control group, in which the rats received an intraperitoneal (i.p.) injection of CYP1A2 inhibitor (cimetidine) of $50 \mathrm{mg} / \mathrm{kg} \mathrm{BW}$ at 5 days prior to the oral administration of water the same as in (a); (d) CYP2E1 inhibitor control group, in which the rats received an i.p. injection of CYP2E1 inhibitor (trans1,2 -dichloroethylene) of $100 \mathrm{mg} / \mathrm{kg} \mathrm{BW}$ at $2.5 \mathrm{~h}$ prior to the oral administration of water the same as in (a); (e) CYP1A2 inhibitor and PMR group, in which the rats received an i.p. injection of cimetidine of $50 \mathrm{mg} / \mathrm{kg} \mathrm{BW}$ at 5 days prior to the oral administration of PMR the same as in (b); (f) CYP2E1 inhibitor and PMR group, in which the rats received an i.p. injection of trans-1,2-dichloroethylene of $100 \mathrm{mg} / \mathrm{kg} \mathrm{BW}$ at $2.5 \mathrm{~h}$ prior to the oral administration of PMR the same as in (b). All rats were kept in separate cages under standard conditions (room temperature of $22-27^{\circ} \mathrm{C}$, relative humidity of $40 \%-70 \%, 12 \mathrm{~h}$ light/dark cycle). They had free access to water and a commercial diet before the treatment and during the treatment interval. All animal studies were performed according to the Guide for the Care and Use of Laboratory Animals of Beijing University of Chinese Medicine. The animal study protocols were approved by the China Animal Care and Use Committee.

2.5. Time Selection to Detect Rat Serum Transaminases in One-Time Treatment of CYP1A2 and CYP2E1 Inhibitor and $P M R$. According to Section 2.4, rats were i.p. injected with CYP1A2 inhibitor, cimetidine, at $50 \mathrm{mg} / \mathrm{kg}$ BW 5 days prior to PMR treatment for (c) and (e) groups and with CYP2E1 inhibitor, trans-1,2-dichloroethylene, at $100 \mathrm{mg} / \mathrm{kg} \mathrm{BW} 2.5 \mathrm{~h}$ prior to PMR treatment for (d) and (f) groups. Then, the rats in (b), (e), and (f) groups received an oral administration of $\mathrm{PM}$ at $40 \mathrm{~g} / \mathrm{kg} \mathrm{BW}$; besides, (a), (c), and (d) groups received water. The level of serum transaminases ALT and AST of rats was detected at $2 \mathrm{~h}, 4 \mathrm{~h}, 24 \mathrm{~h}, 48 \mathrm{~h}$, and $72 \mathrm{~h}$ after the oral administration of PMR.

2.6. Oral Administration of PMR to Rats with or without CYPs Inhibited. According to the clinical characteristics of hepatotoxicity induced by $\operatorname{PMR}[32,33]$, we designed an animal experiment with prolonged and repeated oral administration of the aqueous extract of PMR treatment as follows (Figure 1). The animal group setting and treatment of CYP1A2 or CYP2E1 inhibitors were the same as described in Section 2.4. After inhibitor treatment, (b), (e), and (f) groups were fed with PMR at $40 \mathrm{~g} / \mathrm{kg} \mathrm{BW}$; other groups were fed with water instead, once a day for 1 week. Then, CYP1A2 and CYP2E1 inhibitor treatments and PMR oral administration were all stopped for 2 weeks. Subsequently, rats were treated with CYP1A2 and CYP2E1 inhibitors as above for the second time and then PMR was orally administered just for 1 day. After that, CYP1A2 and CYP2E1 inhibitor treatments and PMR oral administration were stopped again for 3 weeks, and then CYP1A2 and CYP2E1 inhibitors were given; later, PMR was orally administered for 1 to 3 days. Rats were anesthetized with chloral hydrate $(0.3 \mathrm{~g} / \mathrm{kg}$ i.p.) $2 \mathrm{~h}$ after the last administration of PMR to examine rat serum ALT and AST levels. Then, rats were euthanized overnight to examine hepatic light microscopic changes and activity of CYP enzymes.

2.7. ALT and AST Levels Examination. The serum samples were collected at different times as follows: at day 1, day 3, day 5 , and day 7 during the first week of PMR administration, day 22 (the first day after the 2-week interval), day 37 (one day in a 3-week interval), day 44 (the first day after the 3-week interval), and day 46 (the last day of the PMR treatment). The blood samples from rat orbit were collected and centrifuged at $1000 \times \mathrm{g}$ for $10 \mathrm{~min}$, and the supernatant sera were saved. The levels of both ALT and AST were measured using AU-400 fully automatic biochemical analyzer (Olympus Corp.) in the clinical laboratory of Wang Jing Hospital, China Academy of Chinese Medical Sciences.

2.8. Pathological Analysis. Briefly, livers of rats from all groups were fixed with $10 \%$ formalin and embedded with paraffin. The $5 \mu \mathrm{m}$ thick sections on the slide were stained with hematoxylin and eosin (HE staining). Pathological examinations of livers were performed by two pathologists using an optical microscope.

2.9. Preparation of Liver Microsomes and Measurement of CYPs Activity. Liver microsomes were prepared and the CYP1A2/CYP2E1 activity was measured as described previously [29]. Briefly, $20 \mu \mathrm{L}$ of drug probes ( $25 \mu \mathrm{mol}$ phenacetin and $25 \mu \mathrm{mol}$ chlorzoxazone) was added to a $5 \mathrm{~mL}$ microcentrifuge tube and volatilized to dry. Then, rat liver microsomal protein $(2 \mathrm{mg} / \mathrm{mL})$ and NADPH-generation system were 


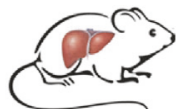

(a)

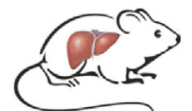

(b)

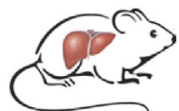

(c)

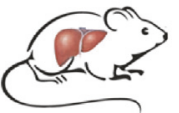

(d)

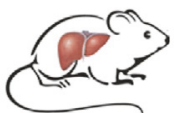

(e)

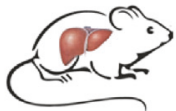

(f)

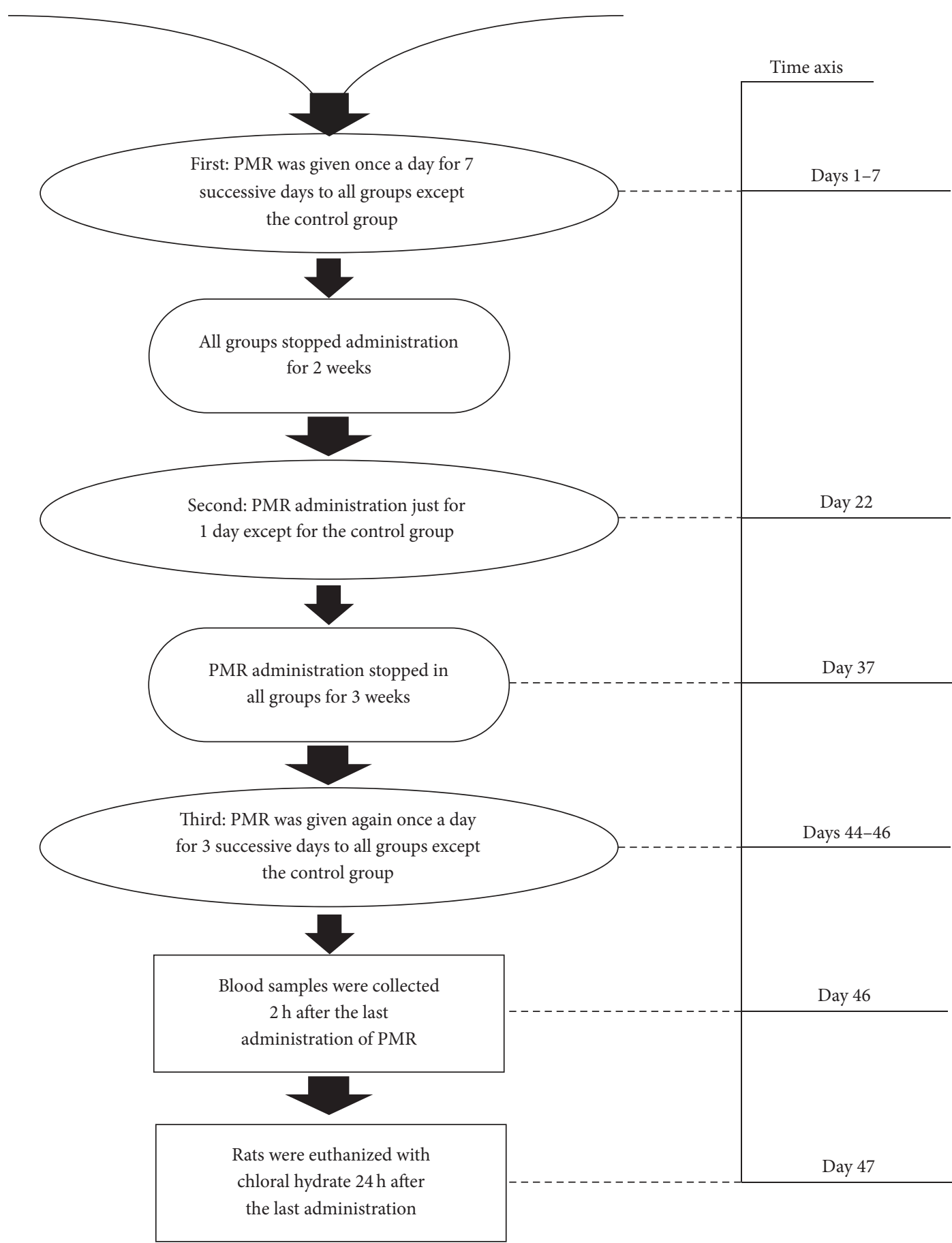

FIGURE 1: Flow chart of the drug administration. (a) Control group: water only. (b) PMR group: PMR only. (c) CYP1A2 inhibitor control group: cimetidine only. (d) CYP2E1 inhibitor control group: trans-1,2-dichloroethylene only. (e) CYP1A2 inhibitor + PMR group: cimetidine and PMR. (f) CYP2E1 inhibitor + PMR group: trans-1,2-dichloroethylene and PMR. 


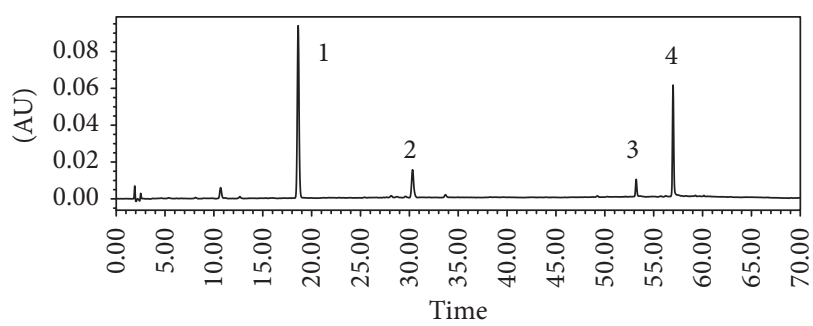

(a)

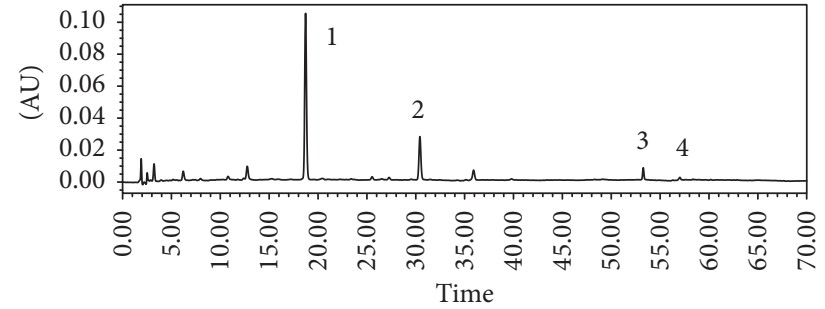

(b)

FIGURE 2: HPLC analysis result of the aqueous extract of PMR. (a) Chromatograms of mixed standard of THSG, EG, emodin, and physcion: 1 for THSG, 2 for EG, 3 for emodin, and 4 for physcion. (b) Chromatogram of the aqueous extract of PMR.

TABLE 1: Contents of the main components in the aqueous extract of PMR.

\begin{tabular}{lcr}
\hline Components & Content $(\mu \mathrm{g} / \mathrm{mL})$ & Percentage of content $(\%)$ \\
\hline THSG & $49.04 \pm 4.73$ & $0.4100 \pm 0.0400$ \\
EG & $6.84 \pm 0.16$ & $0.0570 \pm 0.0010$ \\
Emodin & $0.91 \pm 0.09$ & $0.0080 \pm 0.0008$ \\
Physcion & $0.31 \pm 0.01$ & $0.0030 \pm 0.0001$ \\
\hline
\end{tabular}

added to the tube. The mixture was incubated at $37^{\circ} \mathrm{C}$ for $30 \mathrm{~min}$ and then $600 \mu \mathrm{L}$ ice-cold acetonitrile was added immediately to stop the reaction. Subsequently, $10 \mu \mathrm{L}$ of schisandrin $(0.06 \mathrm{~g} / \mathrm{L})$ was added and the mixture was centrifuged at $10000 \times \mathrm{g}$ for $20 \mathrm{~min}$ at $4^{\circ} \mathrm{C}$ after vortex oscillation. The supernatant was collected from the tube and blown dry with nitrogen, and then $200 \mu \mathrm{L}$ of $\left(\mathrm{NH}_{4}\right)_{2} \mathrm{HPO}_{4}$ was added to redissolve. Finally, the solution was centrifuged at $13000 \times \mathrm{g}$ for $15 \mathrm{~min}$ and $30 \mu \mathrm{L}$ of the supernatant was used for HPLC analysis. The percentage for metabolic elimination of each drug probe was calculated using the following equation:

Probe substrate metabolic elimination percentage

$$
=\frac{(A-B)}{A} \times 100 \%
$$

where $A$ is the quantity of the added probe substrate and $B$ is the quantity of the measured probe substrate.

2.10. Statistical Analysis. The data obtained in the assay was expressed in the form of means $\pm \mathrm{SD}$. One-factor Analysis of Variance in each group was analyzed with SPSS Statistics 17.0. Statistical significance was set at $P<0.05 ; P<0.01$ means extremely significant discrepancy.

\section{Results}

3.1. The Main Components in the Aqueous Extract of PMR. The extraction rate of the aqueous extract was $21.4 \%$. HPLC analysis was adopted to identify the contents of the main components in PMR. In the chromatogram of HPLC, there were four main well-separated chromatographic peaks (Figure 2). They were unambiguously identified as THSG,
EG, emodin, and physcion. The components are shown in Table 1 .

3.2. Treatment with Inhibition of CYP1A2 or CYP2E1 and PMR Administration Significantly Increased Rat ALT and AST Levels at $2 \mathrm{~h}$ after One-Time Oral Administration of PMR. To determine the optimum time for detecting the serum transaminase level after oral administration of PMR, the ALT and AST levels at $2 \mathrm{~h}, 4 \mathrm{~h}, 24 \mathrm{~h}, 48 \mathrm{~h}$, and $72 \mathrm{~h}$ after oral administration of PMR were assayed (Figure 3).

As shown in Figure 3, the administration of PMR along with CYP1A2 inhibitor, cimetidine, or CYP2E1 inhibitor, trans-1,2-dichloroethylene, in rats significantly increased the serum transaminases levels within $24 \mathrm{~h}$. Both ALT and AST levels were increased at $2 \mathrm{~h}$ after PMR administration (CYP1A2 inhibitor + PMR group compared with control group, PMR group, and CYP1A2 inhibitor control group, $P<0.05$; CYP2E1 inhibitor + PMR group compared with control group, PMR group, and CYP2E1 inhibitor control group, $P<0.05)$. The AST level kept elevated at $4 \mathrm{~h}$ after PMR administration (CYP1A2 inhibitor + PMR group compared with control group, PMR group, and CYP1A2 inhibitor control group, $P<0.05$; CYP2E1 inhibitor + PMR group compared with control group, PMR group, and CYP2E1 inhibitor control group, $P<0.05)$ and ALT level kept elevated at $24 \mathrm{~h}$ after PMR administration (CYP1A2 inhibitor + PMR group compared with control group, PMR group, and CYP1A2 inhibitor control group, $P<0.05$; CYP2E1 inhibitor + PMR group compared with control group, PMR group, and CYP2E1 inhibitor control group, $P<0.05)$. ALT or AST levels in all groups showed no significant differences after $48 \mathrm{~h}$. As the rat serum ALT and AST levels significantly increased in the experimental groups, which was detected at $2 \mathrm{~h}$ after administration of PMR, we choose $2 \mathrm{~h}$ after administration of 


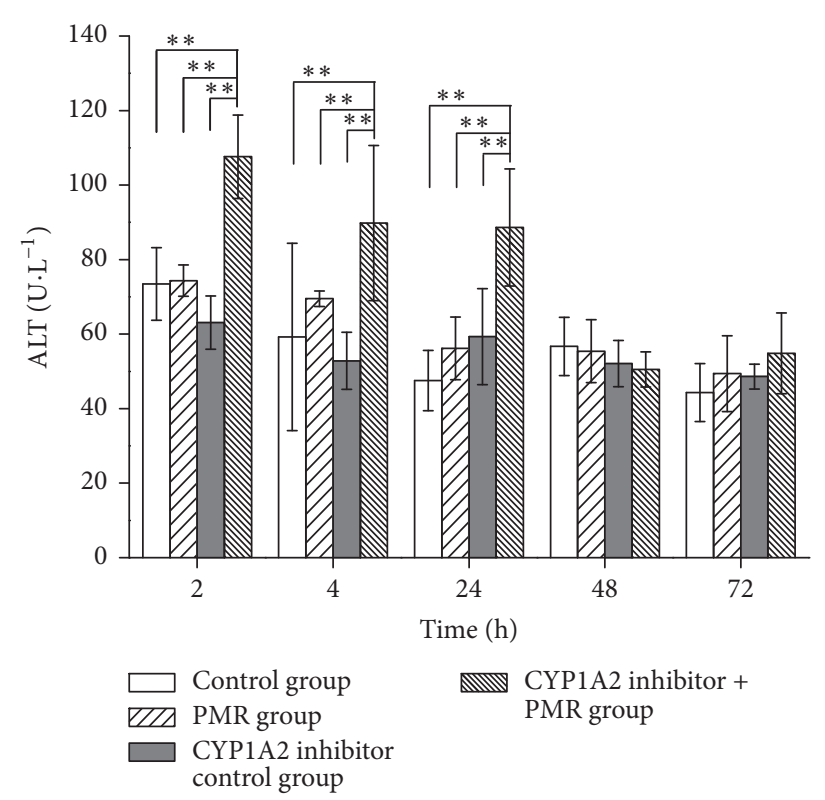

(a)

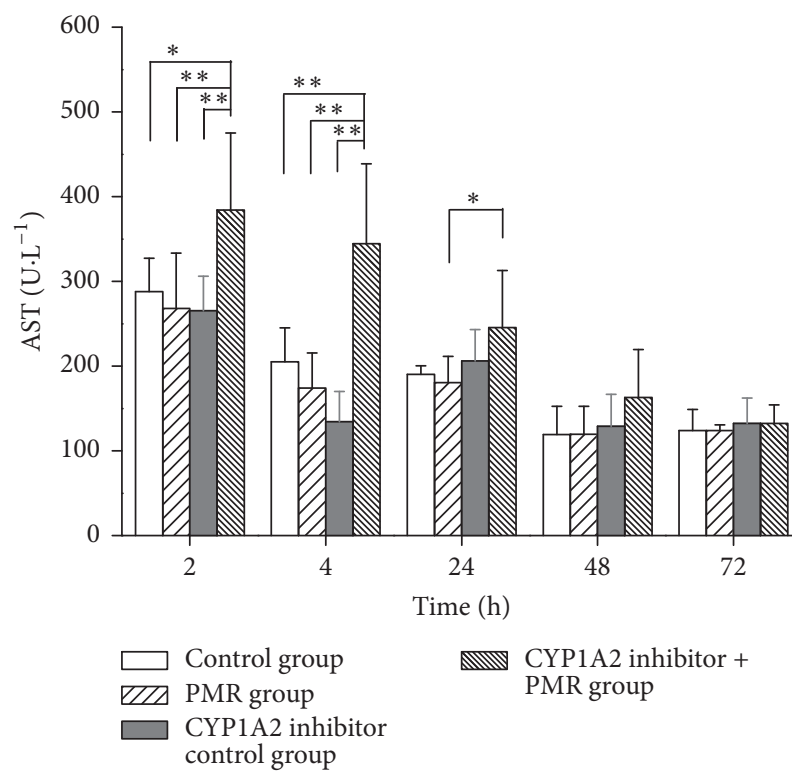

(c)

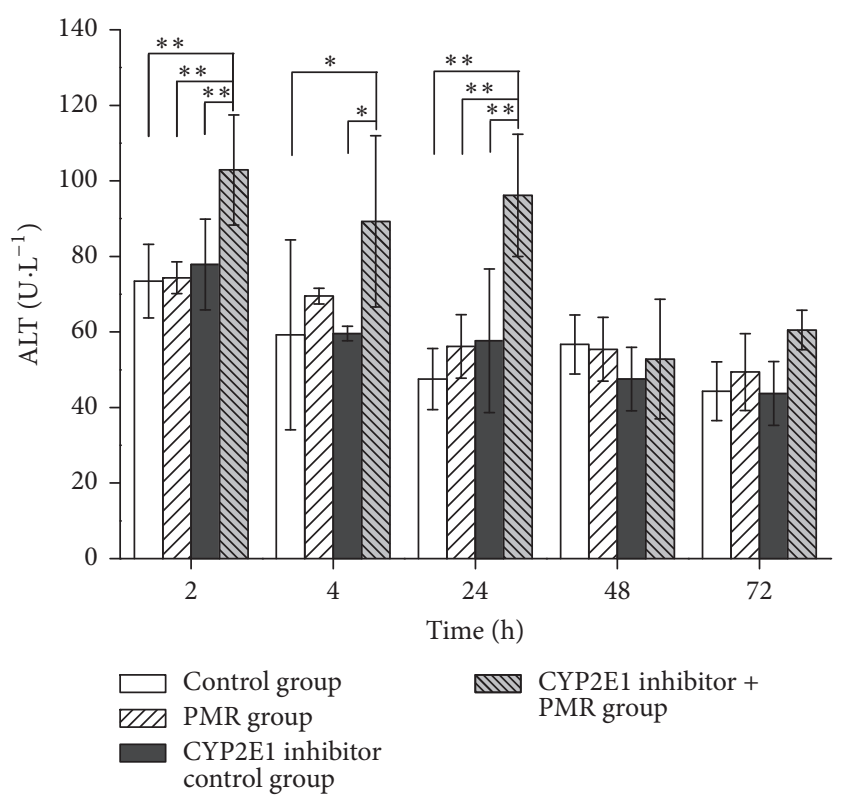

(b)

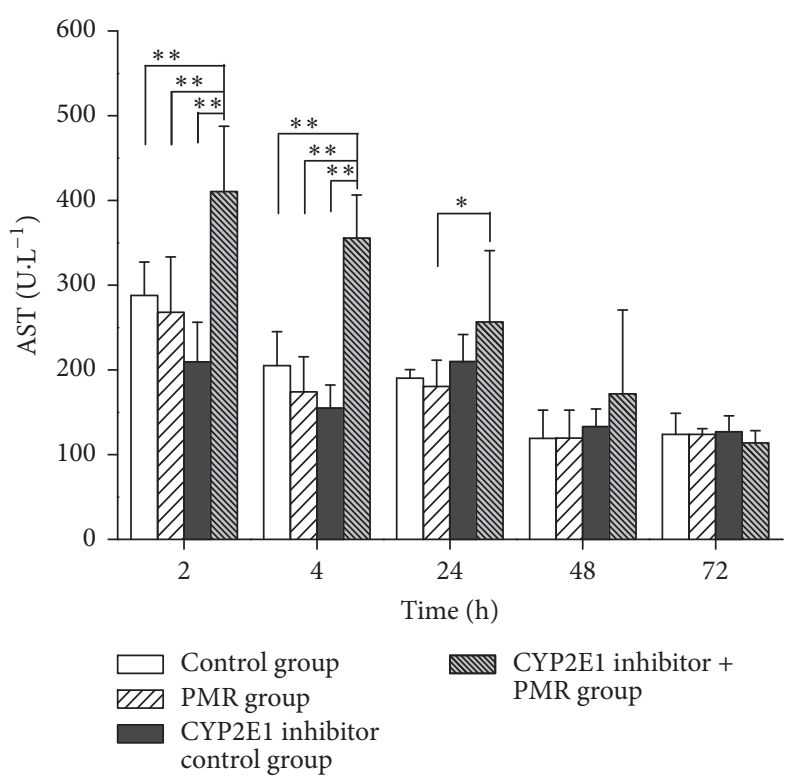

(d)

Figure 3: The rat serum transaminases levels assayed at $2 \mathrm{~h}, 4 \mathrm{~h}, 24 \mathrm{~h}, 48 \mathrm{~h}$, and $72 \mathrm{~h}$ after oral administration of PMR $(\bar{x} \pm s, n=5)$. (a) and (b) for ALT level; (c) and (d) for AST level; (a) and (c) for CYP1A2 inhibited group; (b) and (d) for CYP2E1 inhibited group. *Significant difference compared with other groups, $P<0.05 .{ }^{* *}$ Extremely significant difference compared with other groups, $P<0.01$.

PMR as the time point for detecting the serum transaminase activity in the following experiments.

\subsection{Rat Serum ALT and AST Levels Significantly Increased} after Prolonged and Repeated Administration of PMR. Rat serum ALT and AST levels in all groups were assayed at $2 \mathrm{~h}$ after administration of PMR as described in Section 2.6. As shown in Figure 4, rat serum ALT and AST levels significantly increased when CYP1A2 or CYP2E1 activities were inhibited before PMR administration $(P<0.01)$. Rat serum ALT and AST levels significantly increased in combination group with administration of PMR and CYP1A2 or CYP2E1 inhibitor compared with all control groups at $1 \mathrm{~d}$, $3 \mathrm{~d}, 5 \mathrm{~d}, 7 \mathrm{~d}$, and $22 \mathrm{~d}(P<0.05)$, whereas PMR control groups and CYP inhibitor control groups showed no significant increase, which indicated that PMR could induce liver injury in CYP1A2 or CYP2E1 inhibited rats. In order to eliminate false positives, ALT and AST levels among all groups in the interval days (at $37 \mathrm{~d}$ from the experiment start, e.g., in the middle of 3-week interval days, Figure 1) were tested, which showed no significant group difference. After the 3-week interval, the rats were treated with PMR again and then serum 


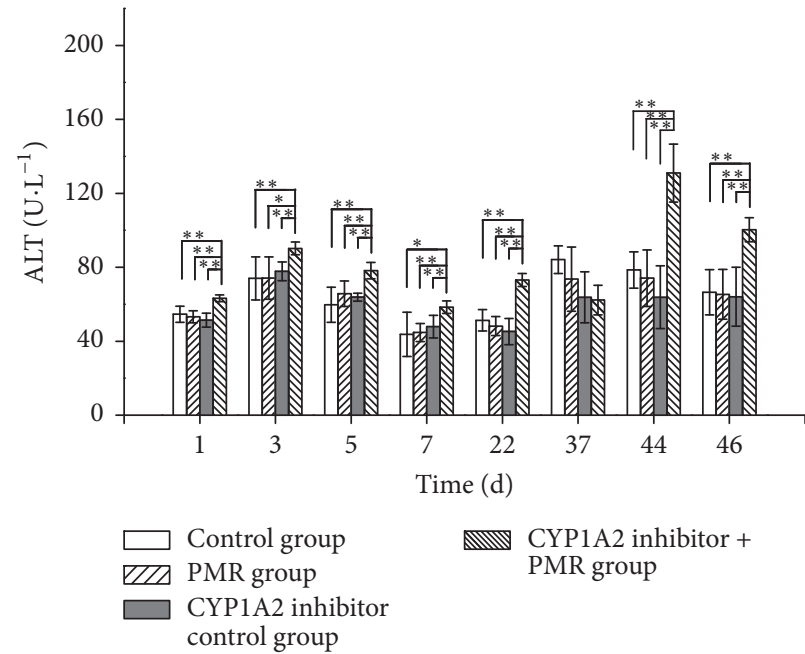

(a)

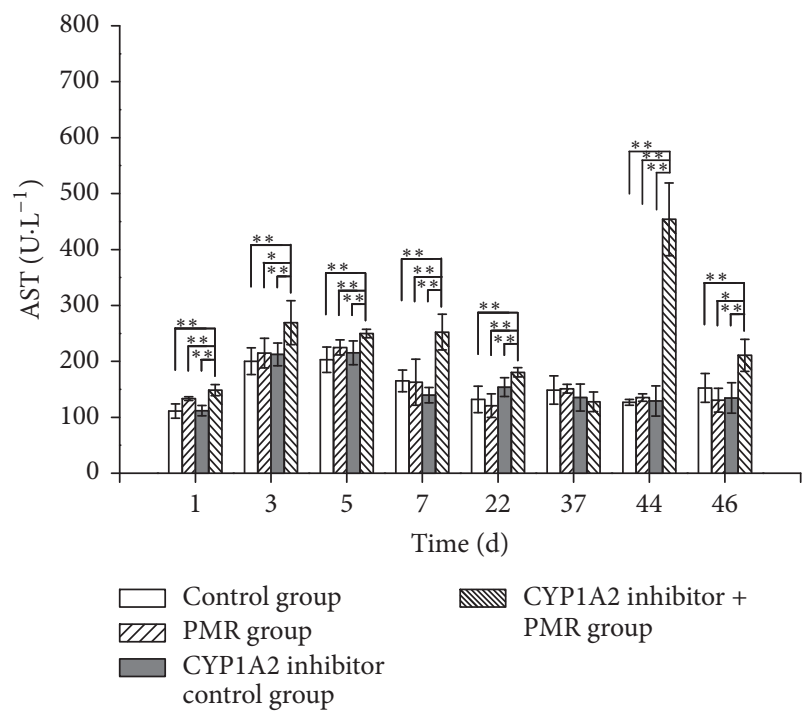

(c)

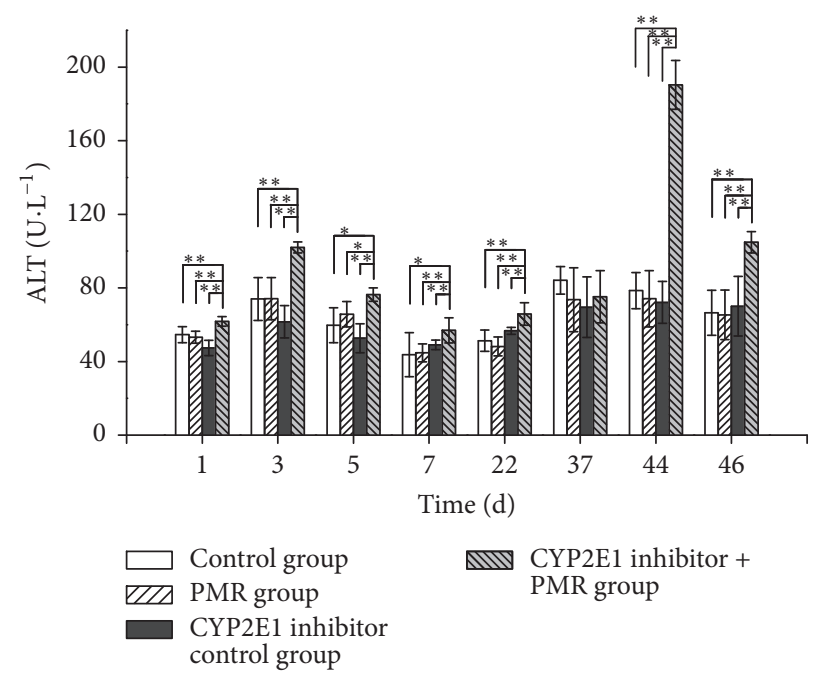

(b)

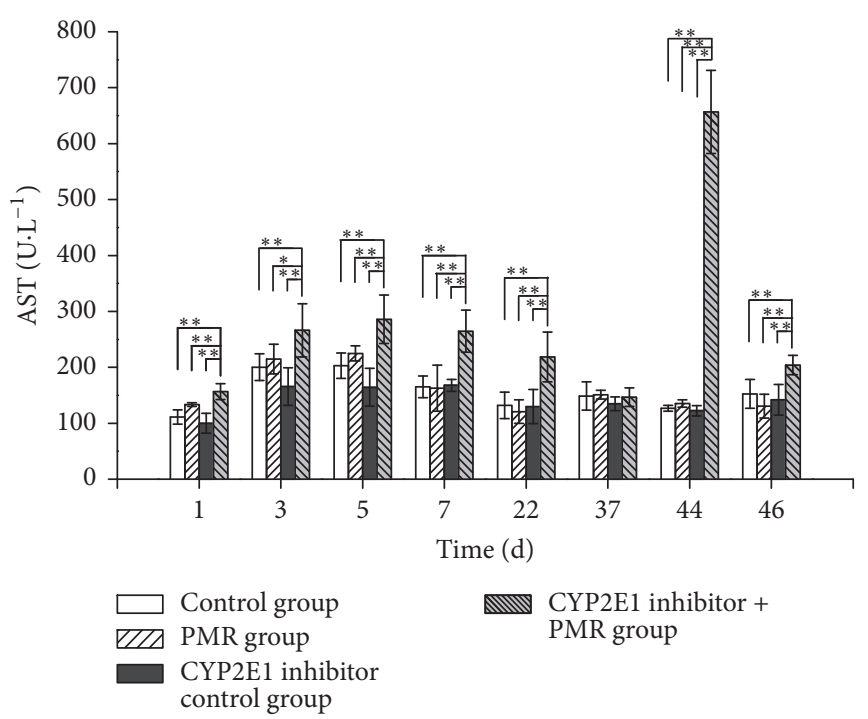

(d)

Figure 4: The measurements of transaminases levels at $1 \mathrm{~d}, 3 \mathrm{~d}, 5 \mathrm{~d}, 7 \mathrm{~d}, 22 \mathrm{~d}, 44 \mathrm{~d}$, and $46 \mathrm{~d}$ after prolonged and repeated oral administration of PMR: oral administration of PMR to rats for 1-7 d, $1 \mathrm{~d}$ after $2 \mathrm{w}$ stop (interval), $1-3 \mathrm{~d}$ for $3 \mathrm{w}$ stop, and $37 \mathrm{~d}$ for transaminases level during oral administration interval ( $\bar{x} \pm s, n=5$ ). (a) and (b) for ALT level. (c) and (d) for AST level. ${ }^{*}$ Significant difference compared with other groups, $P<0.05 .{ }^{* *}$ Extremely significant difference compared with other groups, $P<0.01$.

ALT and AST were assayed; ALT and AST levels extremely significantly increased $(P<0.01)$ at $44 \mathrm{~d}$ and $46 \mathrm{~d}$, whereas ALT and AST levels increased nearly twofold compared to all control groups at $44 \mathrm{~d}$ which showed more severe liver damage.

\subsection{Moderate Liver Injury Occurred in Rats after Administra-} tion of PMR and Inhibitor of CYP1A2 or CYP2E1. Rat liver pathological observation is shown in Figure 5; compared with the control group (a), PMR group (b), CYP1A2 inhibitor control group (c), and CYP2E1 inhibitor group (d), CYP1A2 inhibitor + PMR group (e) showed hepatic sinusoid moderate expansion and congestion in the portal tract with some cell karyopyknosis that is an early stage of apoptosis, while CYP2E1 inhibitor + PMR group (f) showed cytoplasmic hydropic change in hepatocytes and hepatic sinusoid moderate expansion in the portal tract.

3.5. The Activity of CYP1A2 and CYP2E1 in Rat Liver after Prolonged and Repeated Oral Administration of PMR. The activities of CYP1A2 and CYP2E1 in the rat liver from all groups were assayed using percentage of metabolic elimination of two probe substrates, phenacetin and chlorzoxazone, respectively (Sections 2.6 and 2.9). Compared with the control group, the activity of CYP1A2 enzyme was significantly decreased in PMR group $(P<0.01)$, CYP1A2 inhibitor 


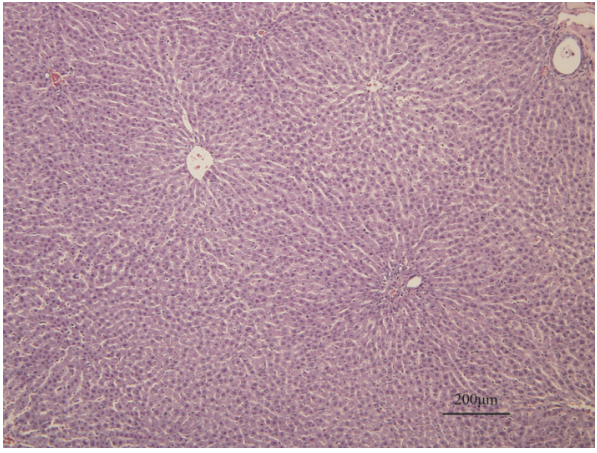

(a)

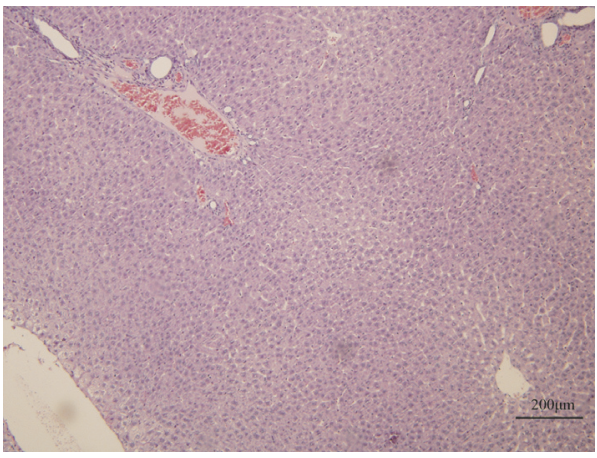

(c)

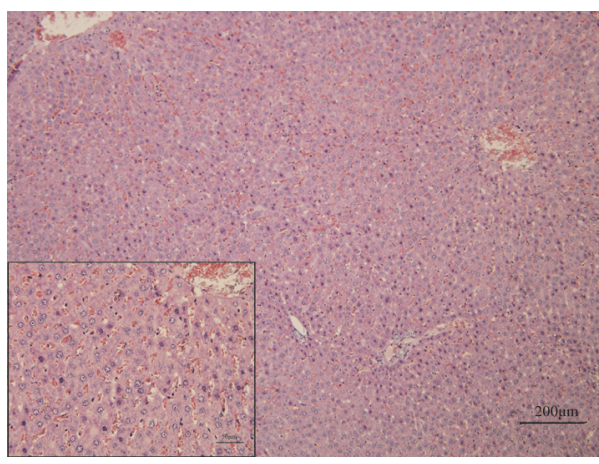

(e)

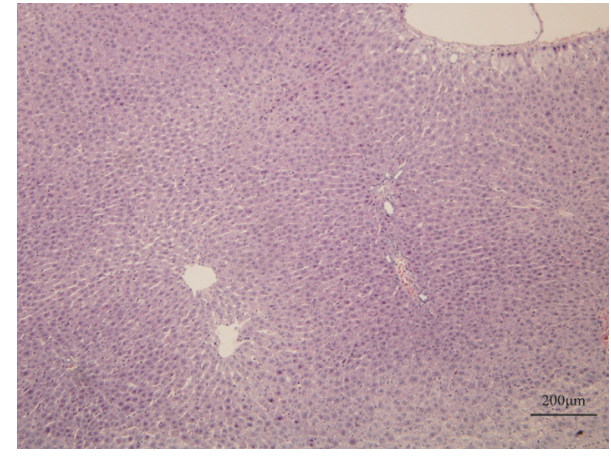

(b)

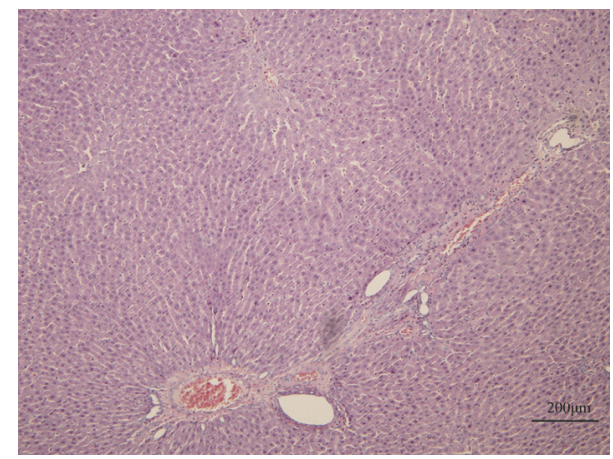

(d)

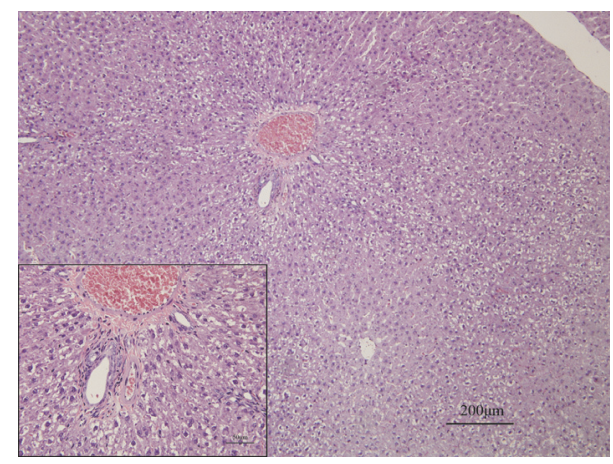

(f)

FIGURE 5: Typical histopathological section photographs of rat hepatic light microscopic changes after administration of PMR and inhibitor of CYP1A2 or CYP2E1. Sections of rat livers from all groups were stained with HE and examined under light microscope (Olympus BX51). (a) Control group; (b) PMR group; (c) CYP1A2 inhibitor control group; (d) CYP2E1 inhibitor control group; (e) CYP1A2 inhibitor and PMR group; (f) CYP2E1 inhibitor and PMR group. The enlarged panels in (e) and (f) indicate the details of pathologic changes.

control group $(P<0.05)$, and CYP1A2 + PMR inhibitor group $(P<0.01)$, whereas the activity of CYP2E1 enzyme was significantly decreased in PMR group $(P<0.05)$, CYP2E1 inhibitor group $(P<0.05)$, and CYP2E1 + PMR inhibitor group $(P<0.05)$ (Figure 6$)$. The results demonstrated that oral administration of $\mathrm{PMR}$ could reduce activities of both CYP1A2 and CYP2E1, while CYP1A2 or CYP2E1 inhibitors had specific inhibition to CYP1A2 or CYP2E1 activities, respectively, in rat liver. CYP1A2 and CYP2E1 enzyme activities were significantly inhibited in CYP1A2 inhibitor + PMR group and CYP2E1 inhibitor + PMR group, respectively.

\section{Discussions}

The current work showed that oral administration of the aqueous extract of PMR did not affect rat serum ALT or AST levels significantly even at dosage of $40 \mathrm{~g} / \mathrm{kg} \mathrm{BW}$ that is 400 -fold the clinical dosage according to the current Chinese Pharmacopoeia. This is consistent with our earlier report [34]. However, rats treated with liver cytochrome P450 enzyme CYP1A2 or CYP2E1 inhibitor with the same dosage of aqueous extract of PMR showed significantly elevated serum transaminase levels. Liver histologic observation revealed that the hepatocytes of experimental groups 


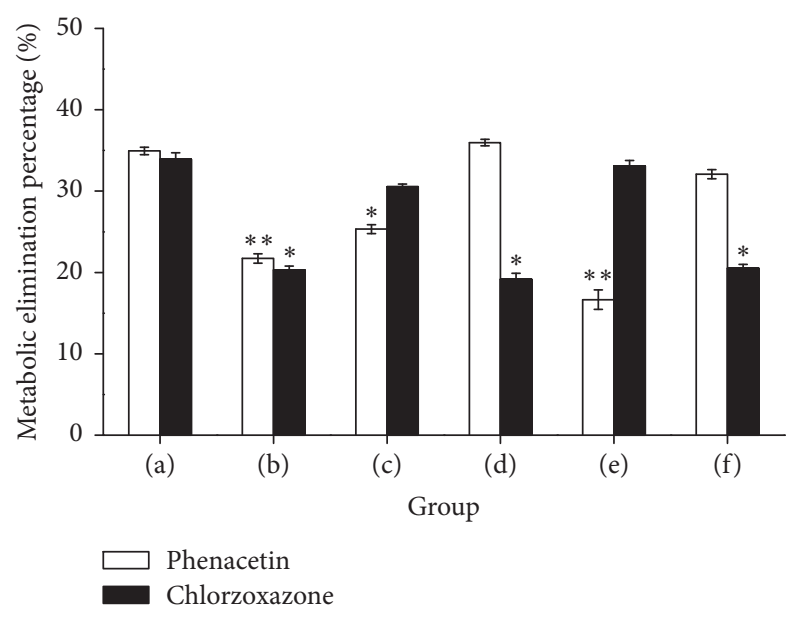

FIGURE 6: Metabolic elimination percentage of two probe substrates, phenacetin (for CYP1A2) and chlorzoxazone (for CYP2E1). (a) Control group; (b) PMR group; (c) CYP1A2 inhibitor control group; (d) CYP2E1 inhibitor control group; (e) CYP1A2 inhibitor and PMR group; (f) CYP2E1 inhibitor and PMR group (shown as mean SD, $n=5 ;{ }^{*}$ significant difference compared with the control group, $P<0.05 ;{ }^{* *}$ extremely significant difference compared with the control group, $P<0.01$ ).

(CYP1A2 inhibitor + PMR or CYP2E1 inhibitor + PMR) showed cytoplasmic hydropic change (CYP2E1 inhibited) or hepatocellular karyopyknosis, which is an early stage of apoptosis (CYP1A2 inhibited).

The very important phenomenon is that serum transaminases ALT and AST levels increased more significantly during repeated PMR treatment after 2 interval times. Rat serum ALT and AST levels significantly increased in the group with the administration of PMR and CYP1A2 or CYP2E1 inhibitor compared with all control groups at $1 \mathrm{~d}, 3 \mathrm{~d}, 5 \mathrm{~d}, 7 \mathrm{~d}$, and $22 \mathrm{~d}$, but no greater than twofold, which suggested that PMR related hepatotoxicity that happened in clinic might be due to some people with lower activities of CYP1A2 or CYP2E1 from genetic polymorphism [22, 23]. Actually, there was a clinical report which suggested that PMR induced hepatotoxicity may relate to low activity of CYP1A2 [28]. ALT and AST levels increased nearly twofold in experimental groups (CYP1A2 inhibitor + PMR or CYP2E1 inhibitor + PMR) compared with all control groups at $44 \mathrm{~d}$ in accordance with the features of PMR related liver injury in the clinic which showed that more severe liver damage happened when PMR was taken repeatedly. Rat serum ALT and AST levels were decreasing at $46 \mathrm{~d}$ compared to at $44 \mathrm{~d}$, suggesting that drug resistance may happen. In order to eliminate false-positive results from different groups, ALT and AST levels among all groups in the interval days (at $37 \mathrm{~d}$, in the middle of 3-week interval days) were tested and no significant group difference in all groups was found, which is also in accordance with the features of PMR related hepatotoxicity that happened in the clinic where liver injury can subside after drug withdrawal.

PMR has been used widely as a medicine in the clinic or as nutritional supplements in China and some other countries. Hundreds of acute hepatitis cases related to PMR have been reported from all over the world [35]. According to the relevant research, the main type of liver injury caused by PMR was hepatocellular. One of the clinical studies reported that the main type of liver injury caused by PMR was hepatocellular (77.8\%), cholestatic (5.6\%), and mixed (16.7\%) [11]. Histological findings exhibited fatty change, infiltration of neutrophils, even apoptotic body, bridging necrosis, and fibrosis in the liver [36], consistent with acute hepatitis laboratory data in which ALT and AST levels elevate remarkably in almost all patients, and total bilirubin and alkaline phosphatase elevate in some patients [33]. In our study, rats in groups of CYP1A2 inhibitor + PMR or CYP2E1 inhibitor + PMR were found to show hepatotoxicity such as hepatocellular hydropic change or karyopyknosis, sinusoid moderate expansion, and congestion in the portal tract, plus significantly elevated ALT and AST levels, especially after repeated administration of PMR. Obviously, the histological feature and biochemical data were not completely consistent with clinical data, indicating that low activity of CYP1A2 or CYP2E1 is just one of the important reasons for PMR related liver injury. Further efforts are needed to reveal other influence factors and declare the mechanisms of PMR related hepatotoxicity.

The possible relationship between inhibition of CYPs and the PMR induced hepatotoxicity suggests that metabolism of some components in the aqueous extract of PMR was retarded. Stilbenes, anthraquinones, and lecithin are known to be the main active substances in PMR [37]. HPLC analysis showed that there were four main well-separated chromatographic peaks standing for THSG, EG, emodin, and physcion in the aqueous extract of PMR (Figure 2). Emodin was found to be the main component of anthraquinones in PMR. Previously, we found that emodin in 95\% ethanoleluted extract of PMR exhibits cytotoxicity and causes cell S phase arrest and apoptosis in human liver L02 cells [38]. Similar results were observed by other research groups [39]. It has been shown that anthraquinones can be metabolized by CYPs, and emodin is known to be metabolized by CYP1A2 enzyme [40]. There were in vivo studies that suggested that emodin may induce rat hepatic lesions [41, 42]. But the main chemical constituents of PMR related to hepatotoxicity need further investigation.

The activity assay of CYPs showed that the activity of CYP2E1 or CYP1A2 was significantly inhibited by the aqueous extract of PMR, but CYP2E1 activity was obviously increased in group (e) (CYP1A2 inhibitor + PMR) or CYP1A2 activity was obviously increased in group (f) (CYP2E1 inhibitor + PMR) after prolonged and repeated administration of PMR. The possible explanation could be that the given CYP inhibitors may have nonspecific targets which may influence some undefined activities of CYP450 enzymes isoforms. Besides CYP1A2 and CYP2E1, the genetic polymorphism of other CYP450 enzymes might be related to the PMR induced hepatotoxicity as well, and the clinical PMR related hepatotoxicity may result from multiple genetic polymorphisms of CYP450 enzymes or some epigenetic modification. All the experimental results from rats need to be confirmed with clinical data, which requires us to collaborate closely with clinical doctors in the future. 


\section{Conclusions}

In conclusion, we have shown that serum transaminases ALT and AST were increased significantly and moderate liver injury appeared in liver histopathological observation, after the administration of PMR in CYP1A2 or CYP2E1 inhibited rats; the result suggested that low level activity of CYP1A2 or CYP2E1 from genetic polymorphism among people might be one of the important reasons for the hepatotoxicity induced by PMR in clinical practice.

\section{Conflicts of Interest}

The authors declare that there are no conflicts of interest regarding the publication of this paper.

\section{Authors' Contributions}

Deng-Ke Li, Jing Chen, and Zhen-Zhen Ge contributed equally to this study.

\section{Acknowledgments}

This work was financially supported by grants from the National Natural Science Foundation of China (no. 81473418) and Beijing University of Chinese Medicine Dongzhimen Hospital Collaborative Innovation Cooperation Project (no. 2016-DZM111-ZY008). The authors are grateful to Dr. Qing Wang and Dr. Hsin-sheng Yang (University of Kentucky, USA) for their critical reading of the manuscript.

\section{References}

[1] National Pharmacopoeia Committee, SP. Pharmacopoeia of the People's Republic of China, People's Medical Publishing House, Beijing, China, 2015.

[2] L. Lin, B. Ni, H. Lin et al., "Traditional usages, botany, phytochemistry, pharmacology and toxicology of Polygonum multiflorum Thunb.: a review," Journal of Ethnopharmacology, vol. 159, pp. 158-183, 2015.

[3] M. N. Han, J. M. Lu, G. Y. Zhang, J. Yu, and R. H. Zhao, "Mechanistic studies on the use of polygonum multiflorum for the treatment of hair graying," BioMed Research International, vol. 2015, Article ID 651048, 8 pages, 2015.

[4] L. Liu, L. Li, L. Zhao et al., "Effects of 2,3,5,4'-tetrahydroxystilbene-2-O- $\beta$-D-glucoside on learning and memory abilities of rats with chronic cerebral ischemia," Chinese Journal of Pharmacology and Toxicology, vol. 22, no. 2, pp. 108-115, 2008.

[5] W. S. Chen, J. P. Xu, L. Li et al., "Studies on nootropic activity and mechanism of emodin-8-O- $\beta$-D-glucopyranoside," Chinese Traditional and Herbal Drugs, vol. 32, no. 1, pp. 39-41, 2001.

[6] R. Zhai, L. S. Lv, and B. Q. Jin, "Hypolipidemic effect of the polysaccharide from polygonum multiflorum," Food \& Machinery, vol. 26, no. 5, pp. 87-101, 2010.

[7] Y. Zhu, Y.-G. Li, J.-B. Wang et al., "Causes, features, and outcomes of drug-induced liver injury in 69 children from China," Gut and Liver, vol. 9, no. 4, pp. 525-533, 2015.

[8] M. Furukawa, S. Kasajima, Y. Nakamura et al., "Toxic hepatitis induced by Show-Wu-Pian, a Chinese herbal preparation," Internal Medicine, vol. 49, no. 15, pp. 1537-1540, 2010.
[9] C. Li, C. Tu, D. Gao et al., "Metabolomic study on idiosyncratic liver injury induced by different extracts of polygonum multiflorum in rats integrated with pattern recognition and enriched pathways analysis," Frontiers Pharmacology, vol. 07, no. 483, 2016.

[10] G. Danan and R. Teschke, "RUCAM in drug and herb induced liver injury: the update," International Journal of Molecular Sciences, vol. 17, no. 1, article 14, 2015.

[11] J. Wang, Z. Ma, M. Niu et al., "Evidence chain-based causality identification in herb-induced liver injury: exemplification of a well-known liver-restorative herb Polygonum multiflorum," Frontiers of Medicine, vol. 9, no. 4, pp. 457-467, 2015.

[12] X. J. Wang, A. H. Zhang, Y. Han et al., "Urine metabolomics analysis for biomarker discovery and detection of jaundice syndrome in patients with liver disease," Mol Cell Proteomics, vol. 11, no. 8, pp. 370-380, 2012.

[13] D. Larrey and S. Faure, "Herbal medicine hepatotoxicity: a new step with development of specific biomarkers," Journal of Hepatology, vol. 54, no. 4, pp. 599-601, 2011.

[14] R. Teschke and A. Eickhoff, "Herbal hepatotoxicity in traditional and modern medicine: actual key issues and new encouraging steps," Frontiers in Pharmacology, vol. 6, no. 27, 2011.

[15] Z. X. Sun and L. Zhang, "Liver damage related to Polygonummultiflorum and its preparation: domestic literature review and analysis," Adverse Drug Reactions Journal, vol. 12, no. 1, pp. 2630, 2010.

[16] A. Corsini and M. Bortolini, "Drug-induced liver injury: the role of drug metabolism and transport," The Journal of Clinical Pharmacology, vol. 53, no. 5, pp. 463-474, 2012.

[17] A. Ortega-Alonso, C. Stephens, M. I. Lucena, and R. J. Andrade, "Case Characterization, clinical features and risk factors in drug-induced liver injury," International Journal of Molecular Sciences, vol. 17, no. 5, pp. 10-3390, 2016.

[18] T. J. Urban, A. K. Daly, and G. P. Aithal, "Genetic basis of drug-induced liver injury: present and future," Seminars in Liver Disease, vol. 34, no. 2, pp. 123-133, 2014.

[19] S. Guaoua, I. Ratbi, F. Z. A. Laarabi et al., "Distribution of allelic and genotypic frequencies of NAT2 and CYP2E1 variants in Moroccan population," BMC Genetics, vol. 15, p. 156, 2014.

[20] N. Singla, D. Gupta, N. Birbian, and J. Singh, "Association of NAT2, GST and CYP2E1 polymorphisms and anti-tuberculosis drug-induced hepatotoxicity," Tuberculosis, vol. 94, no. 3, pp. 293-298, 2014.

[21] T. Shimada, H. Yamazaki, M. Mimura, Y. Inui, and F. P. Guengerich, "Interindividual variations in human liver cytochrome P-450 enzymes involved in the oxidation of drug," Journal of Pharmacology and Experimental Therapeutics, vol. 270, no. 1, pp. 414-423, 1994.

[22] X. He and S. Feng, "Role of metabolic enzymes P450 (CYP) on activating procarcinogen and their polymorphisms on the risk of cancers," Current Drug Metabolism, vol. 16, no. 10, pp. 850863, 2015.

[23] V. Carrière, F. Berthou, S. Baird, C. Belloc, P. Beaune, and I. De Waziers, "Human cytochrome P450 2E1 (CYP2E1): from genotype to phenotype," Pharmacogenetics, vol. 6, no. 3, pp. 203-211, 1996.

[24] X. M. Han, X. P. Chen, Q. N. Wu, C. H. Jiang, and H. H. Zhou, "G-2964A and C734A genetic polymorphisms of CYP1A2 in Chinese population," Acta Pharmacologica Sinica, vol. 21, no. 11, pp. 1031-1034, 2000. 
[25] M. Kot and W. A. Daniel, "Caffeine as a marker substrate for testing cytochrome P450 activity in human and rat," Pharmacological Reports, vol. 60, no. 6, pp. 789-797, 2008.

[26] M. Martignoni, G. M. Groothuis, and R. d. Kanter, "Species differences between mouse, rat, dog, monkey and human CYPmediated drug metabolism, inhibition and induction," Expert Opinion on Drug Metabolism \& Toxicology, vol. 2, no. 6, pp. 875894, 2006.

[27] M. Umeno, O. W. McBride, C. S. Yang, H. V. Gelboin, and F. J. Gonzalez, "Human ethanol-inducible P450IIE1: complete gene sequence, promoter characterization, chromosome mapping, and cDNA-directed expression," Biochemistry, vol. 27, no. 25, pp. 9006-9013, 1988.

[28] K. F. Ma, X. G. Zhang, and H. Y. Jia, “CYP1A2 polymorphism in Chinese patients with acute liver injury induced by Polygonum multiflorum," Genetics and Molecular Research, vol. 13, no. 3, pp. 5637-5643, 2014.

[29] H. Li, H. L. Yang, D. K. Li et al., "Study on inhibitory effect of water extract of Polygonum multiflorum on CYP1A2 and CYP2E1 enzymatic activities and mRNA expressions in rat liver," China Journal of Chinese Materia Medica, vol. 40, no. 7, pp. 1370-1375, 2015.

[30] Z. T. Liang, H. B. Chen, Z. L. Yu, and Z. Z. Zhao, "Comparison of raw and processed Radix Polygoni Multiflori (Heshouwu) by high performance liquid chromatography and mass spectrometry," Chinese Medicine, vol. 5, no. 29, 2010.

[31] C. Zhang, R. C. Zhang, and Z. X. Sun, "Study on the hepatoxicity of polygoni multiflori radix and polygoni multiflori rodix praeparata in rats," Journal of Chinese Medicinal Materials, vol. 36, no. 9, pp. 1416-1419, 2013.

[32] X. Li, C. Qu, Q. He et al., "Acute hepatitis induced by a Chinese herbal product Qibao Meiran Wan: a case study," International Journal of Clinical and Experimental Medicine, vol. 8, no. 7, pp. 11624-11627, 2015.

[33] H. Dong, D. Slain, J. Cheng, W. Ma, and W. Liang, "Eighteen cases of liver injury following ingestion of Polygonum multiflorum," Complementary Therapies in Medicine, vol. 22, no. 1, pp. 70-74, 2014.

[34] C. Zhang, R. C. Zhang, and Z. X. Sun, "Study on the hepatoxicity of polygonimultiflori radix and polygoni multiflorI rodix praeparata in rats," China Journal of Chinese Materia Medica, vol. 36, no. 9, pp. 1416-1419, 2013.

[35] X. Lei, J. Chen, J. Ren et al., "Liver damage associated with Polygonum multiflorum thunb.: a systematic review of case reports and case series," Evidence-Based Complementary and Alternative Medicine, vol. 2015, Article ID 459749, 9 pages, 2015.

[36] K. A. Jung, H. J. Min, S. S. Yoo et al., "Drug-induced liver injury: twenty five cases of acute hepatitis following ingestion of Polygonum multiflorum thunb," Gut and Liver, vol. 5, no. 4, pp. 493-499, 2011.

[37] G.-A. Bounda and Y. Feng, "Review of clinical studies of Polygonum multiflorum Thunb. and its isolated bioactive compounds," Pharmacognosy Research, vol. 7, no. 3, pp. 225-236, 2015.

[38] R.-C. Zhang, B. Liu, Z.-X. Sun, and D.-Y. Xu, "Effects of extract of Polygonum multiflorum on cell cycle arrest and apoptosis of human liver cell line L02," Journal of Chinese Integrative Medicine, vol. 8, no. 6, pp. 554-561, 2010.

[39] J. Yu, J. Xie, X.-J. Mao et al., "Hepatoxicity of major constituents and extractions of radix polygoni multiflori and radix polygoni multiflori praeparata," Journal of Ethnopharmacology, vol. 137, no. 3, pp. 1291-1299, 2011.
[40] S. O. Mueller, H. Stopper, and W. Dekant, "Biotransformation of the anthraquinones emodin and chrysophanol by cytochrome P450 enzymes: bioactivation to genotoxic metabolites," Drug Metabolism and Disposition, vol. 26, no. 6, pp. 540-546, 1998.

[41] C. Tu, D. Gao, X. Li et al., "Inflammatory stress potentiates emodin-induced liver injury in rats," Frontiers in Pharmacology, vol. 6, no. 233, 2015.

[42] J. Ma, L. Zheng, Y.-S. He, and H.-J. Li, "Hepatotoxic assessment of Polygoni Multiflori Radix extract and toxicokinetic study of stilbene glucoside and anthraquinones in rats," Journal of Ethnopharmacology, vol. 162, pp. 61-68, 2015. 


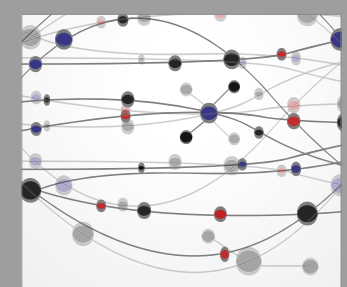

The Scientific World Journal
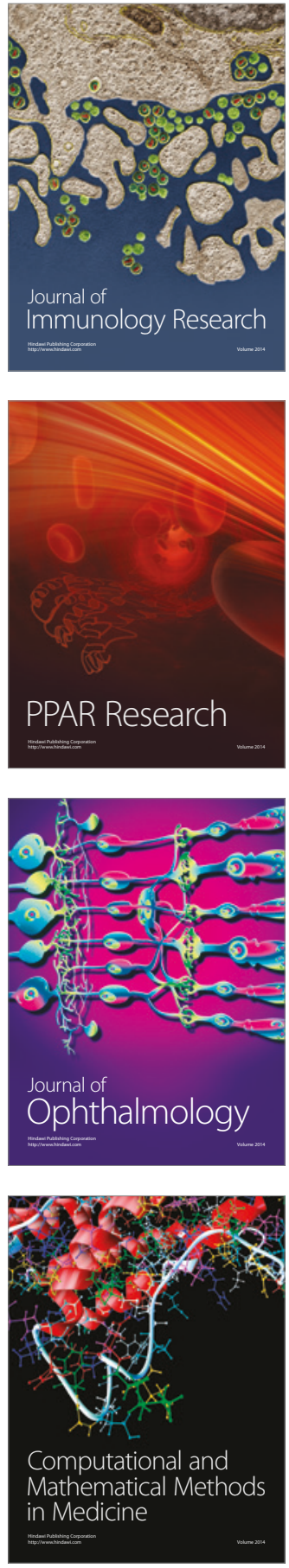

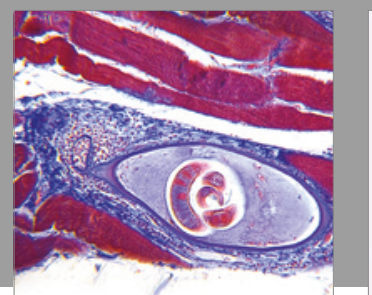

Gastroenterology Research and Practice
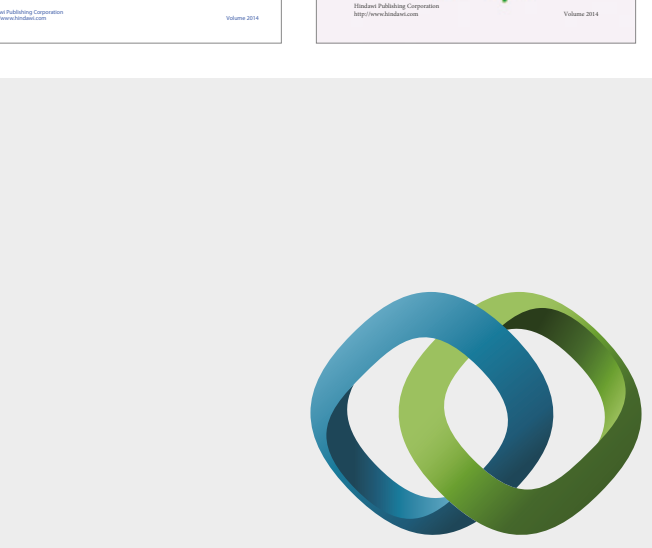

\section{Hindawi}

Submit your manuscripts at

https://www.hindawi.com
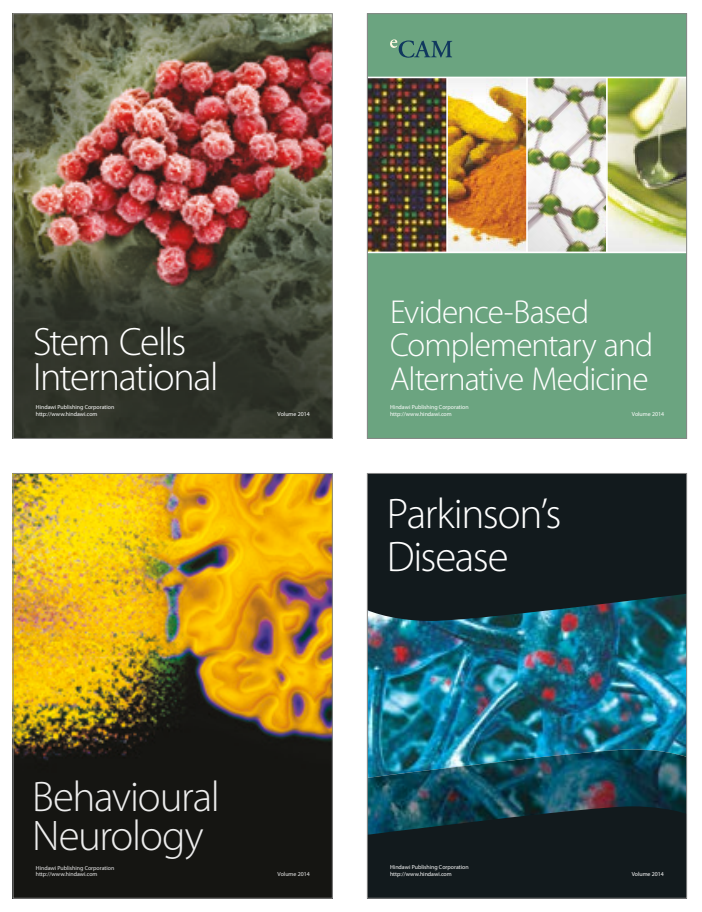
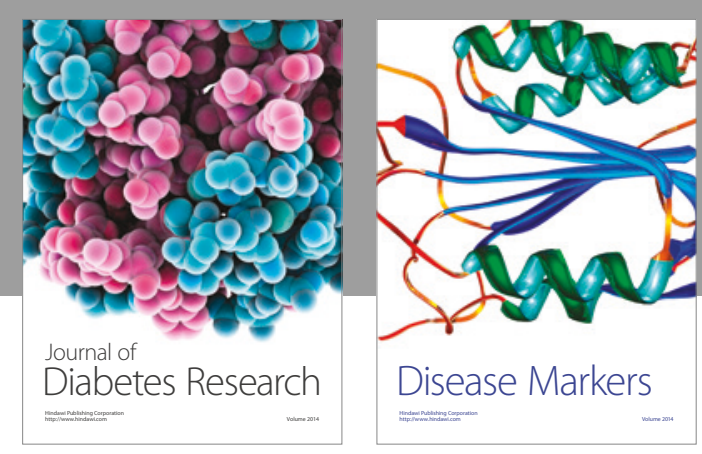

Disease Markers
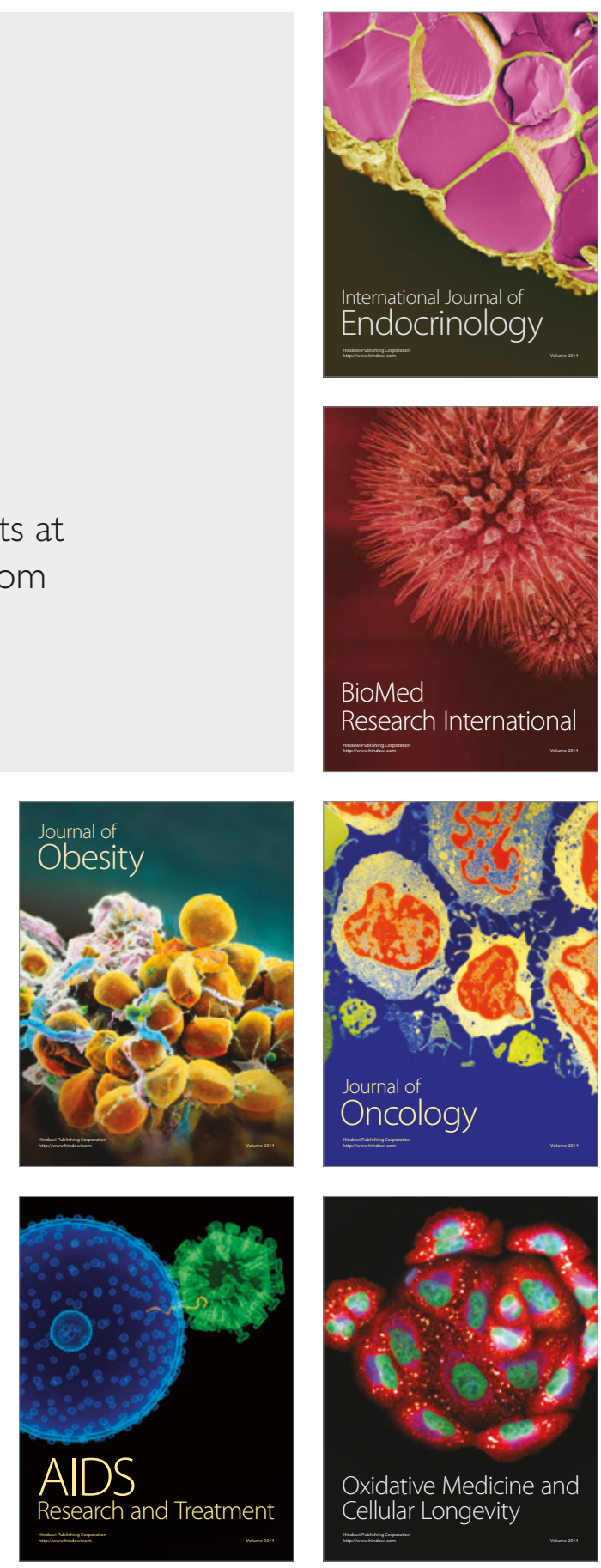